\title{
Usefulness of Ultrasonography-Assisted Closed Reduction for Nasal Fracture under Local Anesthesia
}

\author{
Dae-Hyun Kim, \\ Kyung-Sik Kim \\ Department of Plastic and Reconstructive \\ Surgery, Myong-Ji Hospital, Goyang, Korea
}

No potential conflict of interest relevant to this article was reported.

\begin{abstract}
Closed reduction is the treatment of choice for most nasal bone fractures. In this technique, the nasal bone cannot be directly visualized, proper reduction is confirmed by palpation of the bony contour. This confirmation-via-palpation is in most cases too uncomfortable or painful for patients, and this is the reason why most closed reductions of nasal bone fractures are performed under general anesthesia. Recently, ultrasonography has been adopted as a useful diagnostic method and operative adjunct. In this report, we report the use of ultrasonography as a means to provide palpation-less confirmation of proper reduction, which in turn allows for nasal bone reduction under local anesthesia.
\end{abstract}

Keywords: Nasal bone / Fracture, bone / Ultrasonography

\section{INTRODUCTION}

The nasal bone is a very common site of facial bone fractures because of its prominent morphology and centrality. Mistaken diagnosis or inadequate reduction can lead to nasal deformity and/or other complications. Hence, prompt diagnosis of bone fracture and confirmation of proper reduction is very important [1].

Traditionally, conventional radiography was used for the evaluation of nasal fractures, and computed tomography has been the gold standard. More recently, however, ultrasound is finding increasing use for nasal bone fracture [2-4]. Most reports relate to diagnostic accuracy and few authors have reported the use of ultrasound as an intraoperative instrument [5]

In most cases, nasal bone fractures are reduced under general anesthesia [6]. However, local anesthesia may be more preferable over general anesthesia because of physiologic factors (e.g., several

\section{Correspondence: Kyung Sik Kim}

Department of Plastic and Reconstructive Surgery, Myong-Ji Hospital, 55

Hwasu-ro 14beon-gil, Deokyang-gu, Goyang 10475, Korea

E-mail:kskimps@mjh.or.kr

Received August 24, 2015 / Revised September 9, 2015 / Accepted September 10, 2015 cardiopulmonary disease or unstable vital sign) or because of patient preference.

Generally, local anesthesia is safer but is not as frequently used for nasal bone fractures because of the pain and discomfort associated with extraneous manipulation and palpation of the fractured site needed to confirm proper reduction. In this report, we introduce an ultrasonography-assisted nasal reduction technique which allows for nasal bone reduction under local anesthesia.

\section{IDEA}

The probe used for the ultrasonography exam was 9L-D, a 2-8 MHz linear transducer (LOGIQ E9, GE healthcare, Milwaukee, WI, USA). Preoperatively, ultrasound is used to identify the fracture site in longitudinal and transverse scans (Fig. 1A) and to correlate with CT images (Fig. 1B).

The fracture site was anesthetized with $2 \%$ lidocaine $(1: 100,000$ epinephrine) injected around the anterior ethmoid nerve along the upper border of septum, vestibular roof, and the periosteum. For vasoconstriction, the nasal cavity was packed with gauze 
soaked in $1 \mathrm{mg} / \mathrm{mL}$ epinephrine solution (Bosmin, Je Il Pharm., Seoul, Korea)

Once the patient was prepped and the operative site properly blocked, a sterile plastic cover was secured onto the ultrasound probe. The operator held the ultrasound probe in the non-dominant hand and manipulated the elevator in the right hand (Fig. 2).
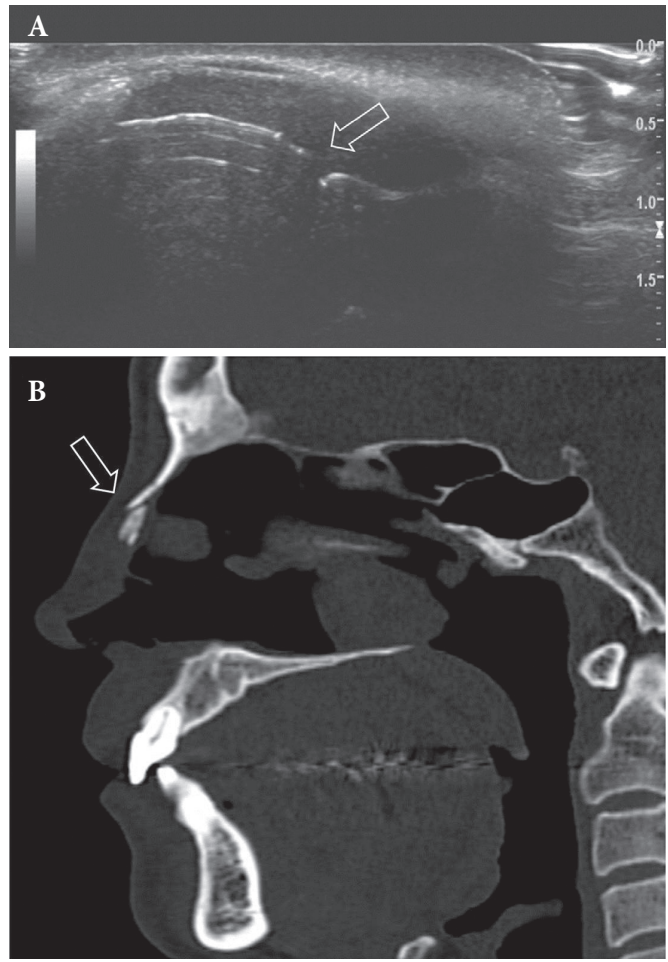

Fig. 1. (A) Preoperative ultrasonography demonstrates cortical discontinuity (arrow) of the nasal bone in the longitudinal plane. (B) The ultrasound image correlates well to computed tomography findings (sagittal view)

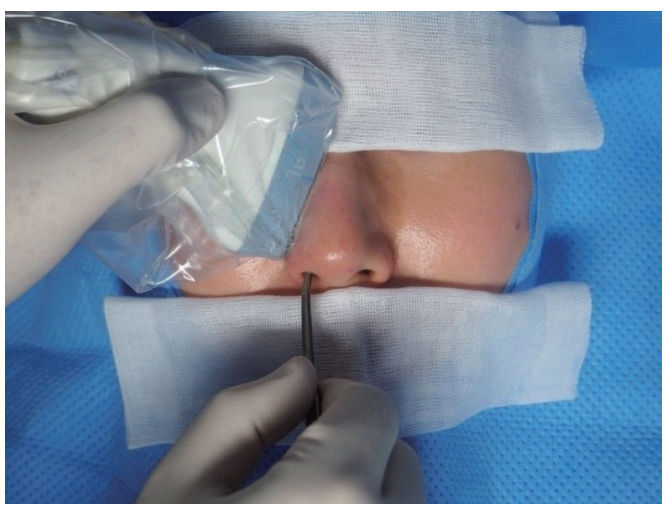

Fig. 2. The probe is held in the non-dominant hand, and the nasal bone is manipulated with the elevator held in the right hand. The probe is oriented longitudinally along the dorsum.
Under the longitudinal view, depressed nasal fractures were reduced by placing a Cushing elevator within the ipsilateral nasal cavity and lifting the fractured bone in the supero-anterior direction. This maneuver was repeated until nasal bone reduction could be confirmed in both longitudinal (Fig. 3) and transverse (Fig. 4) views.

Upon ultrasonographic confirmation of successful reduction, the epinephrine-soaked gauze was removed. The nasal cavity was re-packed with polyacetylvinyl (Merocel ${ }^{\circledR}$, Medtronic Xomed, Jacksonville FL, USA), and the nasal dorsum was dressed with Thermo-splint. The packing was removed after 3 days, and the splint was removed after 3 weeks.

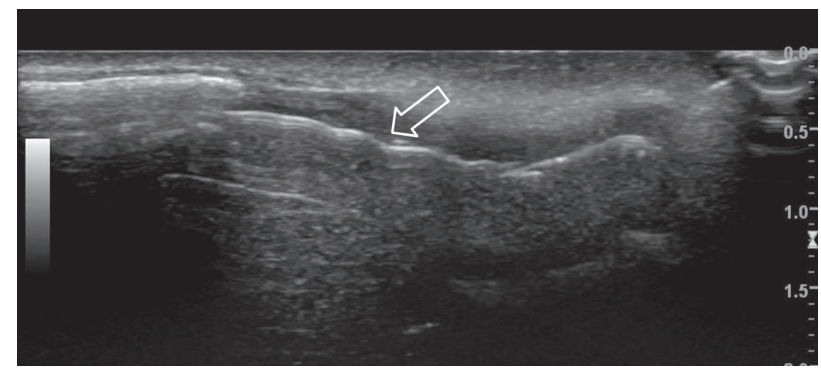

Fig. 3. Longitudinal ultrasonography demonstrates nasal bone in corrected alignment (arrow)
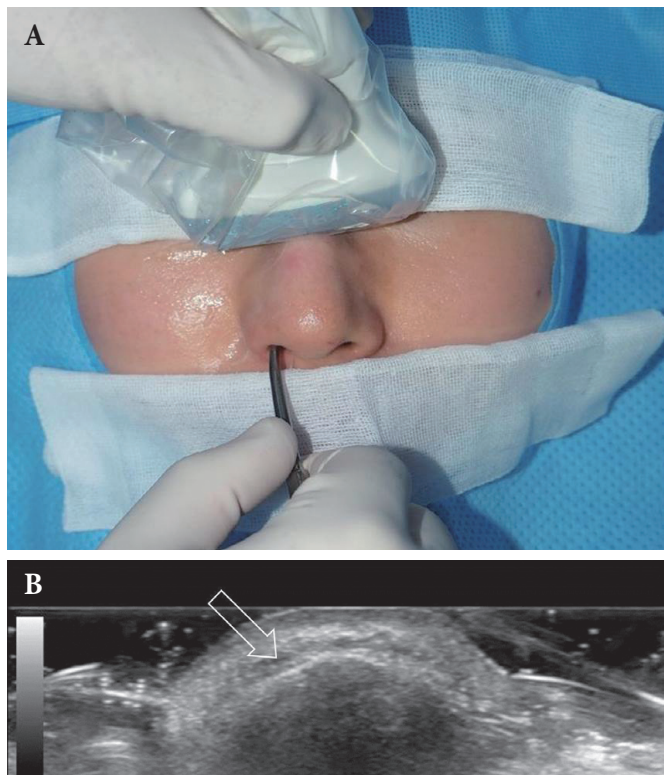

Fig. 4. (A) The ultrasound probe is held a transverse orientation over the dorsum. (B) Transverse ultrasonography demonstrates symmetric contour without displacement (arrow) after closed reduction of the nasal bone. 
At our institution, this ultrasound-guided technique was used for every nasal bone reduction performed under local anesthesia ( $\mathrm{n}=21)$ between January 2014 and June 2015. During this same period, the mean operative time was 25 minutes for ultrasoundguided reduction under local anesthesia versus 55 minutes for conventional reduction under general anesthesia. Also, the reduction results were satisfactory by visual inspection.

\section{DISCUSSION}

Closed reduction is the treatment of choice in nasal bone fractures and the procedure is often performed under general anesthesia. Under local anesthesia, closed reduction is performed under visual inspection and palpation. However, accurate evaluation of nasal bone reduction can be difficult. Because of the soft-tissue swelling and pre-existing asymmetry, visual inspection is often unreliable. Though palpation can be extremely helpful, patients often express discomfort or overt pain from manipulating the fracture site [7].

In this context, intraoperative ultrasonography can decrease several undesirable aspects of local anesthesia. Direct visualization decreases the amount of guesswork involved in confirmation of nasal bone reduction. This consequently decreases over-manipulatioin of the fracture site and operative time.

The longitudinal orientation of the probe to the dorsum reveals sagittal view of the nasal tissue, and the elevator is oriented parallel to this plane. This allows direct visualization of the bone being manipulated by the elevator. The transverse scan is useful in evaluating the symmetricity and contour of the nasal bones.

This technique has advantages, because the reducted nasal bone can be directly visualized on real-time ultrasound and can decrease unnecessary time to palpate and inspect the nose. Also, it can reduce the operator's and patient's anxiety during and after the procedure. Consequently, it can facilitate effective procedure under local anesthesia.

The nasal bone fracture is a common presentation for craniofacial trauma, and nasal bone reduction is generally considered a simple procedure. However, inaccurate reduction can result in nasal deformity that is difficult to correct, and accurate diagnosis and appropriate management is very important.

Ultrasonography is not necessary in all nasal bone fracture patients, but it may be useful in decreasing unnecessary manipulation of the fractured site for patients undergoing local anesthesia reduction. Further refinements in the technique may allow for bedside reductions in the future.

\section{REFERENCES}

1. Park CH, Joung HH, Lee JH, Hong SM. Usefulness of ultrasonography in the treatment of nasal bone fractures. J Trauma 2009;67:1323-6.

2. Friedrich RE, Heiland M, Bartel-Friedrich S. Potentials of ultrasound in the diagnosis of midfacial fractures. Clin Oral Investig 2003;7: 226-9.

3. Jank S, Emshoff R, Etzelsdorfer M, Strobl H, Nicasi A, Norer B. Ultrasound versus computed tomography in the imaging of orbital floor fractures. J Oral Maxillofac Surg 2004;62:150-4.

4. Hong HS, Cha JG, Paik SH, Park SJ, Park JS, Kim DH, et al. High-resolution sonography for nasal fracture in children. AJR Am J Roentgenol 2007;188:W86-92.

5. Abu-Samra M, Selmi G, Mansy H, Agha M. Role of intra-operative ultrasound-guided reduction of nasal bone fracture in patient satisfaction and patient nasal profile (a randomized clinical trial). Eur Arch Otorhinolaryngol 2011;268:541-6.

6. Verwoerd CD. Present day treatment of nasal fractures: closed versus open reduction. Facial Plast Surg 1992;8:220-3.

7. Yabe T, Tsuda T, Hirose S, Ozawa T, Kawai K. Comparison of ultrasonography-assisted closed reduction with conventional closed reduction for the treatment of acute nasal fractures. J Plast Reconstr Aesthet Surg 2014;67:1387-92. 